Résumés des conférences et travaux

\title{
Histoire et codicologie du livre manuscrit arabe
}

\author{
Nuria de Castilla
}

\section{(2) OpenEdition Journals}

\section{Édition électronique}

URL : https://journals.openedition.org/ashp/3512

DOI : $10.4000 /$ ashp.3512

ISSN : 1969-6310

Éditeur

Publications de l'École Pratique des Hautes Études

\section{Édition imprimée}

Date de publication : 1 septembre 2020

Pagination : $54-57$

ISSN : 0766-0677

\section{Référence électronique}

Nuria de Castilla, "Histoire et codicologie du livre manuscrit arabe », Annuaire de l'École pratique des hautes études (EPHE), Section des sciences historiques et philologiques [En ligne], 151 | 2020, mis en ligne le 09 juillet 2020, consulté le 06 juillet 2021. URL : http://journals.openedition.org/ashp/3512 DOI : https://doi.org/10.4000/ashp.3512 


\title{
HISTOIRE ET CODICOLOGIE DU LIVRE MANUSCRIT ARABE
}

\author{
Directrice d'études : $\mathrm{M}^{\mathrm{me}}$ Nuria DE CAstilla
}

Programme de l'année 2017-2018 : Les manuscrits dans l'Occident musulman.

Le cours 2017-2018 a été consacré à l'étude d'une sélection de manuscrits arabes conservés à la bibliothèque de San Lorenzo de El Escorial (Madrid). Cette dernière, qui se trouve placée sous la direction de la communauté de pères augustins, appartient cependant au Patrimoine national (Casa Real). Elle contient dans son état actuel environ 2000 manuscrits arabes parmi lesquels se trouvent ceux qui composaient la bibliothèque des sultans saadiens.

La collection est conservée dans la bibliothèque de l'Escorial à la suite de sa capture par l'Espagne en 1612 sous le règne de Mulay Zaydān (1603-1627); elle constitue une «capsule temporelle » exceptionnellement préservée. Contrairement à d'autres bibliothèques de manuscrits médiévaux ou modernes du monde islamique, son contenu n'a pas été modifié au point de rendre complètement impossible la reconstitution des fonds originaux. Nous ne possédons ni liste ni catalogue précis de la collection de l'Escorial à la fin du XVI ${ }^{\mathrm{e}}$ siècle ou au début du XVII ${ }^{\mathrm{e}}$, mais nous pouvons examiner les livres, retracer leur histoire et identifier ainsi ceux qui se trouvaient sur place avant l'arrivée de la collection saadienne. Cette dernière peut ainsi être exactement définie. Elle reflète les intérêts et les goûts des dirigeants saadiens et de l'élite en général. Cette situation est presque unique dans le monde islamique, à l'exception de l'Empire ottoman; en effet, comme ces collections ont généralement continué jusqu'au $\mathrm{XIX}^{\mathrm{e}}$ ou même au $\mathrm{XX}^{\mathrm{e}}$ siècle à être enrichies par de nouvelles acquisitions ou à perdre des livres plus anciens, il est très difficile de déterminer l'état de leurs collections à un moment donné du passé - à moins qu'un catalogue daté ne fournisse les informations nécessaires. En ce qui concerne les reliures, les artisans locaux ont réparé ou remplacé celles qui étaient en mauvais état au cours des siècles. Dans certains cas, le changement est évident parce que le style et / ou la technique ne correspondent pas à la date du manuscrit, mais dans de nombreux cas, il sera difficile de parvenir à une conclusion, vu que certains outils, par exemple les plaques, ont été utilisés sur de longues périodes.

La bibliothèque saadienne n'est cependant pas complète : certains manuscrits qui sont arrivés à l'Escorial ont été perdus lors d'un incendie en 1671; d'autres volumes ont été laissés au Maroc, probablement pendant la fuite de Mulay Zaydān, et sont maintenant dispersés dans diverses bibliothèques. Il n'est pas surprenant d'en trouver au Maroc, par exemple à la Bibliothèque nationale du Royaume du Maroc à Rabat. Mais certains ont été trouvés dans d'autres pays : une copie du Kashshāf d'al-Zamakhshari avec la marque de possession d'Aḥmad al-Manșūr sur son premier feuillet fait partie des collections de la bibliothèque de l'université de Cambridge (Add. 815). 
Les manuscrits fournissent de nouvelles informations sur l'histoire de la bibliothèque elle-même. Comme on pouvait s'y attendre, beaucoup portent une marque manuscrite indiquant qu'ils appartenaient à Zaydān, le fils d'Aḥmad al-Manșūr, qui a perdu sa bibliothèque à la suite du soulèvement dirigé par Ibn abī Mahallī : ce dernier a chassé le sultan de sa capitale de Marrakech en 1612 et l'a forcé à s'embarquer avec sa suite et ses livres, ou du moins une bonne partie d'entre eux, sur un bateau qui les a emportés vers Agadir, un voyage qui s'est avéré fatidique pour la bibliothèque. Il n'est pas surprenant que beaucoup de livres portent également les marques de possession de son père, Aḥmad al-Manșūr, qui était arrivé au pouvoir après la « Bataille des trois rois » en 1578 et s'est employé à s'imposer comme le «calife des savants et le savant des califes » selon une formule inventée par un érudit contemporain, Aḥmad al-Manjūr (mort en 1587).

Aḥmad al-Manșūr tenait à établir sa figure de savant et les sources concernant son règne, notamment la chronique d'al-Fishtalī, en disent long sur ses efforts dans cette direction. Il s'est efforcé de se conformer aux procédures de l'érudition musulmane traditionnelle et certains manuscrits de sa collection ont en fait conservé les licences (ijāza) qu'il a reçues de la part de savants contemporains.

Malgré la perte d'un bon nombre de volumes lors d'un incendie qui a ravagé le monastère de l'Escorial en 1671, cette bibliothèque est demeurée relativement intacte ce qui fait d'elle une des très rares collections de livres manuscrits du monde musulman antérieure au XVII ${ }^{\mathrm{e}}$ siècle qui ait été préservée.

Pendant les conférences, nous avons analysé l'histoire de différentes bibliothèques du monde musulman de manière à apprécier le caractère exceptionnel de celle qui se trouve à l'Escorial. Le noyau principal a été la lecture et l'analyse d'un corpus de paratextes sélectionnés sur des manuscrits de cette collection et regroupant les notes de possession, les différents certificats associés à la transmission du texte, les prix, etc. Cette analyse nous a permis de réfléchir sur les intérêts de lecteurs princiers marocains auX XVI ${ }^{\mathrm{e}}$ et $\mathrm{XVII}{ }^{\mathrm{e}}$ siècles.

En mai 2018, la professeure Sabine Schmidtke (IAS, Princeton) a été invitée par la directrice d'études pour donner une série de conférences intitulée « Pour une reconstruction des bibliothèques de la période médiévale et du début de l'âge moderne dans le monde musulman », dont les enseignements ont complété les contenus de la conférence de la directrice d'études pendant l'année 2017-2018.

Programme de l'année 2018-2019: Les manuscrits arabes de la Bibliothèque nationale d'Espagne.

La Biblioteca Nacional de España (Bibliothèque nationale d'Espagne) abrite une importante collection de manuscrits arabes (900 environ), qui fut cataloguée en partie en 1889 par Francisco Guillén Robles ${ }^{1}$, dont le travail a été complété par Derenbourg

1. Francisco Guillén Robles, Catálogo de los manuscritos árabes existentes en la Biblioteca Nacional de Madrid, Madrid, Imprenta y Fundición de Manuel Tello, 1889. 
$(1904)^{2}$, Dagorn et alii $(1974)^{3}$ et Justel Calabozo $(1980)^{4}$; de son côté, Saavedra attira l'attention sur les manuscrits aljamiados de la BNE dans l'appendice inclus dans son allocution d'entrée à la Real Academia de la Historia (1878) .

En dépit de toutes ces publications, les manuscrits sont en définitive encore assez peu connus, parce que les auteurs cités plus haut n'ont réalisé que des inventaires sommaires, avec des notes plus ou moins étendues sur le contenu mais sans être accompagnées d'une description matérielle ou codicologique qui pourrait nous offrir des données sur la datation et le lieu de copie des différents manuscrits, écrits en arabe ou aljamía - c'est-à-dire une variante de l'espagnol avec des caractéristiques linguistiques spécifiques employée par les mudéjars et les morisques entre les $\mathrm{XV}^{\mathrm{e}}$ et $\mathrm{XVII}^{\mathrm{e}}$ siècles et écrite principalement en caractères arabes, mais aussi à l'occasion en caractères latins. De ce fait, avec l'exception des manuscrits qui possèdent un colophon, le lecteur de ces catalogues n'a aucune idée de la date de copie d'une œuvre donnée ni de l'endroit où cette copie a été réalisée. L'histoire de ce fond est mal documentée. Par voie de conséquence, il est nécessaire d'aller consulter les manuscrits pour vérifier si la copie a été faite au Maghreb ou dans la péninsule Ibérique (dans le cas où elle est en écriture maghrébine) et si elle est d'époque médiévale ou moderne, voire postérieure. Certaines descriptions sont encore plus difficiles à comprendre; sous le numéro LVIII du catalogue de Guillén Robles (p. 27), par exemple, on trouve d'abord le nom de l'auteur, al-Ghazālī, et le titre : «Chemin royal des dévôts vers le paradis » («Camino real de los devotos hacia el Paraíso »). Mais dans la suite de la description, le lecteur apprend qu'il s'agit d'un manuscrit arabe en écriture maghrébine copié en 905 / 1500, comportant 154 feuillets, sans titre ni nom de l'auteur. Guillén Robles ajoute : «Es conocidamente de moriscos » [il est très connu parmi les Morisques], sans donner plus d'explication. Veut-il dire qu'il y a des annotations postérieures morisques? Que l'œuvre a été copiée plusieurs fois au XVI ${ }^{\mathrm{e}}$ siècle? Que le manuscrit a été copié lui-même par un morisque (même si ce terme ne commence à être appliqué aux musulmans qui ont été obligés de se convertir au catholicisme qu'à partir de 1502)?

Les conférences d'histoire et codicologie du livre manuscrit arabe 2018-2019 ont été consacrés à la description et l'étude d'une sélection de manuscrits en caractères arabes conservés à la Bibliothèque nationale d'Espagne à partir de numérisations mises en ligne par cette bibliothèque et de photos prises par la directrice d'études lors de ses investigations à Madrid. Ces conférences ont été le résultat de la recherche en cours menée par la directrice d'études, qui est en train de cataloguer et d'étudier les manuscrits en caractères arabes qui ont probablement circulé dans la péninsule

2. Hartwig Derenbourg, Notes critiques sur les manuscrits arabes de la Bibliothèque nationale de Madrid, Paris, Imprimerie Orientale G. Maurin, 1904.

3. R. Dagorn, Teresa Losada et M. V. Villuendas, « Un nuevo fondo de manuscritos árabes fragmentarios de la Biblioteca Nacional de Madrid», Al-Andalus, XLII, 1 (1977), p. 123-166.

4. Braulio Justel, «Otro nuevo fondo de manuscritos árabes de la biblioteca Nacional de Madrid», Awrâq, 3 (1980), p. 52-59.

5. Eduardo Saavedra, "Índice general de la literatura aljamiada », dans Discursos leídos ante la Real Academia Española el día 20 de diciembre de 1878, Madrid, Imp. de la Compañía de Impresores y Libreros, 1878, p. 103-182. 
Ibérique entre le $\mathrm{XV}^{\mathrm{e}}$ et le début du XVII ${ }^{\mathrm{e}}$ siècle et qui sont conservés à la Bibliothèque nationale d'Espagne. La date de composition ou de copie des manuscrits sélectionnés pour le cours 2018-2019 est variable, ce qui a permis de proposer une définition - et, dans certains cas, une évaluation - des différents aspects matériels de chaque manuscrit et de chaque époque dans le cadre des quatre siècles au cours desquels ont été copiés les manuscrits analysés. De la même manière, en se fondant sur l'étude des contenus des manuscrits, les goûts littéraires des communautés musulmanes de la péninsule Ibérique à la fin du Moyen Âge et au début de l'époque moderne ont été abordés et étudiés.

La directrice d'études a obtenu le financement d'un « séjour de mobilité internationale » $(\mathrm{SMI})$ du CNRS pour travailler à Madrid pendant quatre mois (été-automne 2018) de manière à mener à bien la première phase du travail de catalogage et d'étude de ces fonds. Par ailleurs, deux étudiants Erasmus+ de Turquie (Fatih Sultan Mehmet Vakif University, FSMVU) ont participé à un stage sur des manuscrits arabes de ce même corpus pendant l'été 2019 (juin-août). 